auch der Berufsverband Deutscher Psychologinnen und Psychologen - BDP - den Bachelorstudiengang Business Psychologie an der Fachhochschule G. nicht zu den anerkannten psychologischen Bachelorstudiengängen zählt und die Mitgliedschaft von Absolventen dieses Studiengangs im Berufsverband ablehnt (aktualisiert Dezember 2015).

Die fehlende Kongruenz zwischen dem von der Kl. absolvierten Wirtschaftspsychologie-Bachelorstudiengang und einem grundständigen Psychologiestudiengang wurde durch das ,reguläre“ Masterstudium der Kl. an einer Technischen Universität in dem Fach Psychologie nicht aufgehoben. Insgesamt fehlen - so der Gutachter in der E-mail an die K1. v. 17.2.2014 - in den psychologischen Grundlagenfächern Studienanteile im Umfang von mindestens 40 ECTS. Da ein Semester durchschnittlich 30 ECTS (Lenz, in: Epping, a.a.O., $\$ 7$, Rdnr. 23) umfasst, dürfte - überschlägig geschätzt - die Studienleistung von deutlich mehr als einem Hochschulsemester fehlen.

Selbst wenn man folglich annähme, dass der konsekutive Bachelor- und Masterstudiengang an einer Universität oder gleichgestellten Hochschule im Fach Psychologie für die Psychotherapeutenausbildung nach $\ 5$ Abs. 2 S. 1 Nr. 1 a PsychThG keine zwingende Voraussetzung ist, könnte sich die Kl. aufgrund der aufgezeigten gravierenden Wissenslücken nicht darauf berufen, dass sie mit der Verleihung des Mastergrades an der TU Chemnitz im Fach Psychologie zugleich auch die Anspruchsvoraussetzungen des $\int 5$ Abs. 2 S. Nr. 1a PsychThG erworben hat. Dabei ist der Umstand, dass die TU Chemnitz die Kl. in den Masterstudiengang Psychologie aufgenommen hatte, sicherlich beachtenswert, wenngleich dies für das in den einzelnen Bundesländern für das Gesundheitswesen jeweils zuständige Landesprüfungsamt nicht bindend sein kann. Universitäten sind verpflichtet zu prüfen, ob Absolventen von Bachelorstudiengängen anderer Hochschulen (dazu zählen Universitäten und gleichgestellte Hochschulen sowie Fachhochschulen, vgl. $\$ 2$ Abs. 1 Nrn. 1 und 2 NHG) die fachlichen Voraussetzungen für den Masterstudiengang - ob konsekutiv oder nicht - erfüllen und können bzw. müssen bei freier Kapazität aus Gründen der Freiheit der Berufswahl (Art. 12 Abs. 1 GG) und des Rechts auf Chancengleichheit (Art. 3 Abs. 1 GG) Bewerber ggf. zu dem Masterstudium zulassen (Nds. OVG, Beschl. v. 17.5.2013 - $2 \mathrm{ME}$ $74 / 13$-, juris, Rdnrn. $11 \mathrm{ff}$.). Eine Übereinstimmung mit den Zulassungskriterien $\mathrm{zu}$ dem psychotherapeutischen Ausbildungsgang in $\ 5$ Abs. 2 S. 1 Nr. 1a PsychTHG folgt daraus nicht ohne weiteres, wie bereits aus der Vorgabe erkennbar ist, dass die Abschlussprüfung das Fach Klinische Psychologie einschließen muss, was offenbar nicht zwingend der Fall ist. Ergänzend sei angemerkt, dass die K1. möglicherweise in den Genuss einer Normierungslücke gekommen war. Denn zur Zeit der Aufnahme der Kl. den Masterstudiengang Psychologie an der TU Chemnitz waren dem dortigen Prüfungsausschuss in $\$ 3$ Abs. 1 der Studienordnung für den Zugang ,,anderer Bewerber" keinerlei Vorgaben an die Hand gegeben, während bereits in der Fassung des $\$ 3$ der Studienordnung v. 7.8.2013 die Zugangsvoraussetzungen dahingehend präzisiert worden waren, dass ein ,,inhaltlich gleichwertiger Studiengang“ verlangt wurde.

Jedenfalls liegt es in der Kompetenz des Bekl., die Gleichwertigkeit selbst noch einmal zu überprüfen, da er die $\mathrm{Zu}-$ ständigkeit für die Approbationserteilung und damit auch die Prüfung der Voraussetzungen für eine entsprechende Ausbildung durch den Landesgesetzgeber mit entsprechender Ermächtigung des Bundesgesetzgebers übertragen bekommen hat, \$S 10 Abs. 1 S. 1, 2 Abs. 1 PsychThG i. V. mit PsychTh-APrV i. V. mit $\$ Abs. 1 Nr. 4 ApproZustV (so auch VG Kassel, a.a. O., s. o., Rdnr. 25). Ansprüche auf Gleichheit im Unrecht vermag eine möglicherweise unterschiedliche Zugangspraxis der Landesprüfungsämter, die sachlich nicht begründet ist, nicht $\mathrm{zu}$ begründen (vgl. BVerwG, Beschl. v. 13.12.2013 - 2 B 37.13 -, juris, Rdnr. 9; und v. 4.4.2013 - 2 B 87.12 -, juris, Rdnr. 10, jew. m. w. N.).

[...]

Die Berufung wurde wegen grundsätzlicher Bedeutung zugelassen ( $\$ S 124$ a Abs. 1 S. 1, 124 Abs. 2 Nr. 3 VwGO). [...]

DOI: $10.1007 / \mathrm{s} 00350-016-4465-0$

\section{Anmerkung zu VG Hannover, Urt. v. 17.2.2016 - 5 A 12344/14}

\section{Hermann Plagemann}

1. Die vom VG Hannover in Bezug genommene Entscheidung des VG Kassel ist im Ergebnis bestätigt worden durch ein Urteil des HessVGH ${ }^{1}$. Gegen diese Entscheidung ist die zugelassene Revision zum BVerwG eingelegt worden ${ }^{2}$. Im Revisionsverfahren geht es um die Frage, ob im Lichte des Art. 12 GG die Verwaltungsbehörden ebenso wie die Instanzgerichte befugt sind, die Voraussetzungen für eine weitergehende Ausbildung gem. $\$ 5$ Abs. 2 PsychThG restriktiv auszulegen und damit das Tatbestandsmerkmal „Abschlussprüfung im Studiengang Psychologie“ durch weitere - ungeschriebene - Voraussetzungen zu beschränken - dies auch im Hinblick auf den vom Gesetz sicherlich mit ins Auge gefassten Patientenschutz. Ob aktuell der Schutz der Patienten durch ein konsekutives Studium besser sichergestellt wird als durch den Studiengang, den die Klägerin hier absolviert hat, erscheint auch und gerade deshalb zweifelhaft, weil herkömmlich das Studium der Psychologie außerordentlich vielfältig aufgebaut war. Das Studium der Psychologie führte keineswegs immer zum Beruf des klinischen Psychologen, sondern hatte sehr unterschiedliche Schwerpunkte, zwischen denen die Studenten wählen konnten, ohne dass das sich daran anschließende Diplom Zweifel hinsichtlich der Zulassung zur Ausbildung begründete.

Schließlich ist zu beachten das Grundrecht der Wissenschaftsfreiheit gem. Art. 5 Abs. 3 GG, welches allen staatlichen Stellen aufgibt, die ,Eigenrationalität der Wissenschaft" zu beachten ${ }^{3}$. Wenn eine Hochschule nach abgeschlossenem Studium plus Leistungsnachweisen feststellt, dass die Qualifikation als Master im Fach Psychologie erreicht ist, stellt sich die Frage, wieweit im Nachhinein eine Differenzierung in Betracht kommt.

2. Aktuell wird über eine Reform der Psychotherapeutenausbildung in den politischen Gremien diskutiert. Nach den Vorstellungen des Bundesgesundheitsministeriums und der Bundespsychotherapeutenkammer soll die Ausbildung künftig ein elfsemestriges Hochschulstudium der Psychotherapie incl. Praxissemester auf „Masterniveau“ umfassen, das mit dem Staatsexamen abschließt und eine Approbation vorsieht ${ }^{4}$. Das Bundesgesundheitsministerium hat zur Novellierung im Nov. 2016. „Eckpunkte“ vorgelegt.

1) Hess. VGH, Urt. v. 4.2.2016 - 7 A $983 / 15$-, juris (auch unter Bezug auf Eichelberger, in: Spickhoff [Hrsg.], Medizinrecht, 2. Aufl. 2014, \5 PsychThG, Rdnr. 15).

2) Az.: 3 C 12.16 .

3) S.u.a. BVerfG v. 17.2.2016 - 1 BvL 8/10 -

4) Vgl. dazu u.a. Bühring, DÄBl. 2016, A-854.

Rechtsanwalt Prof. Dr. iur. Hermann Plagemann,

PLAGEMANN RECHTSANWÄLTE Partnerschaft mbB,

Niedenau 13-19, 60325 Frankfurt am Main, Deutschland 